\title{
A Bulk Parameterization of Giant CCN
}

\author{
DAVID B. MECHEM \\ Cooperative Institute for Mesoscale Meteorological Studies, University of Oklahoma, Norman, Oklahoma, and Department of \\ Geography, University of Kansas, Lawrence, Kansas \\ YeFIM L. KogAN \\ Cooperative Institute for Mesoscale Meteorological Studies, University of Oklahoma, Norman, Oklahoma
}

(Manuscript received 24 April 2007, in final form 13 November 2007)

\begin{abstract}
A parameterization for giant cloud condensation nuclei (GCCN), suitable for use in bulk microphysical models, has been developed that uses precise representations of the condensational growth of aerosol particles in the subcloud layer. The formulation employs an observationally based GCCN distribution function and directly observable parameters of GCCN, such as concentration and the shape of the aerosol spectra. The parameterization couples naturally to parameterizations of sea salt flux from the ocean surface. The behavior of the GCCN parameterization in a large eddy simulation (LES) framework is consistent with simulations employing explicit, size-resolving microphysical methods. The parameterization properly represents the sensitivity of cloud, drizzle, turbulence, and radiative properties to changes in GCCN concentration for polluted and clean background $\mathrm{CCN}$ environments.
\end{abstract}

\section{Introduction}

Previous studies have suggested that precipitation by stratocumulus in the marine boundary layer (MBL) can significantly affect boundary layer thermodynamics and energetics, leading to profound changes in cloud amount and internal cloud properties (Paluch and Lenschow 1991; Stevens et al. 1998). Accurately representing the sensitivity of cloud and radiative properties to microphysical processes involving precipitation is important for assessing aerosol indirect effects in largescale models and for correctly forecasting MBL cloud systems on shorter time scales.

The classical model of precipitation formation and growth proceeds through the nucleation of cloud droplets on cloud condensation nuclei $(\mathrm{CCN})$, growth by condensation, and growth by coalescence (Beard and Ochs 1993). The long time scale required for condensational growth of droplets large enough for collection efficiencies to become significant has been a subject of

Corresponding author address: David B. Mechem, Department of Geography, University of Kansas, 1475 Jayhawk Blvd., 213 Lindley Hall, Lawrence, KS 66045-7613.

E-mail: dmechem@ku.edu ongoing investigation for many years. In short, this simple model of nucleation, condensation, and coalescence-the warm rain process-is insufficient to explain the rapid growth of precipitation-sized droplets in observations. The bottleneck in the classical theory is the development of sufficient droplets in the $\sim 20-25$ $\mu \mathrm{m}$ range.

Giant CCN (GCCN; $1<r<10 \mu \mathrm{m}$ ) have been suggested as one mechanism that may serve to "short circuit" the coalescence bottleneck. More precisely, GCCN may be responsible for the development of drizzle embryos over this size range, which could initiate the development of precipitation (Johnson 1982). Studies have shown that sea salt nuclei are often present in concentrations similar to those of drizzle drops, implying they play an important role in drizzle production (O'Dowd et al. 1997). Furthermore, although high $\mathrm{CCN}$ concentrations are typically associated with the suppression of precipitation (Albrecht 1989), the presence of GCCN may in fact encourage the precipitation process in such an environment (Johnson 1982).

Because of their high solubility and large size, sea salt GCCN deliquesce at subsaturated relative humidity (RH) into droplets many times their dry size. However, 
only the smallest of the deliquesced nuclei reach their equilibrium (Kohler) size at $100 \% \mathrm{RH}$ in a short time (i.e., on the order of seconds). Because of the slowness of condensational growth, upon entering the cloud base the size of the larger particles is typically less than equilibrium size. Mordy (1959) assumed that particles smaller than $0.12 \mu \mathrm{m}$ at the cloud base deliquesced to equilibrium radii at $100 \% \mathrm{RH}$, while particles larger than $1.2 \mu \mathrm{m}$ deliquesced to a size corresponding to equilibrium at $90 \% \mathrm{RH}$. Thus, the larger deliquesced GCCN are not "activated" in a Kohler sense yet nevertheless may be of sufficient size to serve as drizzle (coalescence) nuclei when ingested into the cloud layer (Johnson 1982). For example, a 3.5- $\mu$ m sodium chloride $(\mathrm{NaCl})$ nucleus at an equilibrium $\mathrm{RH}$ of $90 \%$ deliquesces to a "wet" nucleus of $20.2 \mu \mathrm{m}$ [see Table 1 in Kogan (1991)], in the range of drop sizes that can initiate coalescence.

Bulk microphysical parameterizations rely on a process termed autoconversion to represent the formation of embryonic drizzle drops by the coalescence of cloud drops. Various formulations of the autoconversion term exist, for example, based on simple ad hoc assumptions (Kessler 1969; Tripoli and Cotton 1980), large eddy simulation (LES) with size-resolving microphysical processes (Khairoutdinov and Kogan 2000, hereafter KK2000) or kinetic potential barrier theory (Liu et al. 2004). Each approach attempts to represent that component of the classical warm rain process associated with the coalescence bottleneck.

Parameterizing the effects of GCCN in bulk microphysical models has largely been neglected. In one recent study, however, Saleeby and Cotton (2004) emphasized the importance of a large cloud droplet mode, which they assumed to have nucleated on GCCN. Here we present a new parameterization of giant $\mathrm{CCN}$ suitable for use in bulk microphysical models alongside any of these commonly employed autoconversion parameterizations. Employing a large eddy simulation framework, we demonstrate that the GCCN parameterization compares favorably to an explicit, size-resolving microphysical representation. The parameterization is able to capture the sensitivity of cloud physical and radiative properties to changes in GCCN concentration for both polluted and clean background $\mathrm{CCN}$ environments.

\section{Formulation of the GCCN parameterization}

The GCCN parameterization is based on first principles and uses an accurate representation of the condensational growth of aerosol particles (CCN wetting) in the subcloud layer. The liquid water content of the deliquesced GCCN is

$$
q_{g}=4 / 3 \pi \rho_{l} \int_{r_{0}}^{\infty} r^{* 3} n\left(r^{*}\right) d r^{*},
$$

where $r^{*}$ is the deliquesced GCCN radius, $n\left(r^{*}\right) d r^{*}$ is the number of GCCN in the interval between $r^{*}$ and $r^{*}+\Delta r^{*}$, and $\rho_{l}$ is the density of liquid water. Based on detailed calculations of nucleation in the subcloud layer performed by Ivanova et al. (1977), the size of the deliquesced nuclei exceed the size of the dry nuclei by a factor of $k$ :

$$
r^{*}=k r_{d}
$$

where

$$
k\left(r_{d}\right)=a w^{-0.12} r_{d}^{0.214},
$$

and $a$ is $5.8\left(\mathrm{~m} \mathrm{~s}^{-1}\right)^{0.12} \mu \mathrm{m}^{-0.214}, w$ is vertical velocity $\left(\mathrm{m} \mathrm{s}^{-1}\right)$, and $r_{d}$ is GCCN dry radius $(\mu \mathrm{m})$. For a characteristic stratocumulus vertical velocity of $0.6 \mathrm{~m} \mathrm{~s}^{-1}$ and GCCN radii from 1 to $10 \mu \mathrm{m}$, the factor $k$ ranges from 6.2 to 3.8. Incorporating Eq. (2) into Eq. (1) gives

$$
q_{g}=4 / 3 \pi \rho_{l} \int_{r_{0}}^{\infty} r_{d}^{3} k\left(r_{d}\right)^{3} n\left(r_{d}\right) d r_{d} .
$$

According to numerous observations by Junge (1972) and others, the aerosol size distribution for $r>0.1 \mu \mathrm{m}$ can be expressed as a power law of the form

$$
n\left(r_{d}\right)=C r_{d}^{-(\alpha+1)}
$$

where $\alpha$ typically varies between 3 and 4 , and the constant $C$ depends on geographical and meteorological conditions. By integrating (5) from a GCCN lower bound of $r_{0}$ up to $\infty, C$ can be expressed as a function of the total concentration of giant nuclei $\left(N_{g}\right)$ and the shape parameter $\alpha$ :

$$
C=N_{g} \alpha r_{0}^{\alpha} .
$$

Here $N_{g}$ and $\alpha$ constitute specification of the GCCN properties.

Bulk microphysical parameterizations traditionally partition the total liquid water into two categories, cloud and rainwater, based on a threshold radius of 20-25 $\mu \mathrm{m}$. The most important effect of GCCN is through their impact on precipitation initiation by providing a source of drizzle embryos, which in the bulk framework falls into the rainwater category.

Although the lower bound of the classical definition of GCCN is $1 \mu \mathrm{m}$, this number is in fact rather arbitrary. In a bulk microphysical framework, the GCCN lower bound should be physically consistent with the threshold between cloud and raindrops. Such a physi- 
cally based lower bound corresponds to dry GCCN particles with radii of $3 \mu \mathrm{m}$ or larger. These particles deliquesce to about $20 \mu \mathrm{m}$ in the subcloud layer and are best able to serve immediately as drizzle embryos once they experience further growth passing through the supersaturated cloud-base region. Droplets nucleated on smaller GCCN require a longer residence time in cloud to grow above the critical $20-\mu \mathrm{m}$ coalescence threshold. The 3- $\mu \mathrm{m}$ GCCN lower bound is also consistent with the tendency in other studies to emphasize the importance of the middle and upper parts of the GCCN range (in addition to the much larger nuclei of the ultragiant category) to the precipitation process (Johnson 1982; Cooper et al. 1997; Feingold et al. 1999; Blyth et al. 2003).

Substituting Eqs. (3), (5), and (6) into Eq. (4), and integrating from a lower bound of $r_{l}$ rather than $r_{0}$ gives

$$
q_{g}=\frac{4}{3} \pi \rho_{l} \int_{r_{l}}^{\infty} r_{d}^{3}\left(a w^{-0.12} r_{d}^{-0.214}\right)^{3} \frac{N_{g} \alpha r_{0}^{\alpha}}{r_{d}^{\alpha+1}} d r_{d}
$$

which reduces to

$$
q_{g}=A N_{g} r_{0}^{\alpha} \frac{\alpha}{\alpha-2.358} r_{l}^{2.358-\alpha},
$$

where $A=4 / 3 \pi \rho_{l} a^{3} w_{0}{ }^{-0.36}$. The physical significance of $r_{l}>r_{0}$ is such that only GCCN with $r>r_{l}$ become embryonic drizzle droplets.

Differentiating Eq. (8) with respect to time gives

$$
\frac{d q_{g}}{d t}=A r_{0}^{\alpha} \frac{\alpha}{\alpha-2.358} r_{l}^{2.358-\alpha} \frac{d N_{g}}{d t},
$$

where $d q_{g} / d t$ is in $\mathrm{g} \mathrm{cm}^{-3} \mathrm{~s}^{-1}$. Dividing by the density of air $\left(\rho_{a}\right)$ transforms the GCCN water content source rate in Eq. (9) into a mixing ratio $\left(\mathrm{g} \mathrm{g}^{-1} \mathrm{~s}^{-1}\right)$ :

$$
\frac{d r_{g}}{d t}=\frac{A r_{0}^{\alpha}}{\rho_{a}} \frac{\alpha}{\alpha-2.358} r_{l}^{2.358-\alpha} \frac{d N_{g}}{d t} .
$$

Assuming $r_{0}=1 \mu \mathrm{m}, r_{l}=3 \mu \mathrm{m}, \alpha=3$, and an air density of $10^{-3} \mathrm{~g} \mathrm{~cm}^{-3}$, the mixing ratio expression becomes

$$
\frac{d r_{g}}{d t}=2.267 \times 10^{-6} \frac{d N_{g}}{d t} .
$$

When grid-mean saturation occurs, it is assumed that all GCCN in that grid become drizzle embryos instantaneously.

\section{Comparison to the bin microphysical approach}

We implemented the GCCN parameterization into the bulk version of the Cooperative Institute for Mesoscale Meteorological Studies (CIMMS) LES and tested it by comparing it with the LES employing explicit, size-resolving microphysics. In the LES framework, the bulk microphysical parameterization of KK2000 was demonstrated to have an accuracy of better than $20 \%$ for the major dynamical and microphysical parameters (see Figs. 7-12 in KK2000). This uncertainty must be taken into account when comparing the differences between the bulk GCCN parameterization and results based on the explicit formulation.

The conservation equation for precipitable water, combining source terms from both KK2000 and the new GCCN parameterization, is

$$
\begin{aligned}
\frac{\partial q_{r}}{\partial t}= & -\frac{\partial u_{i} q_{r}}{\partial x_{i}}+\frac{V_{q r} q_{r}}{\partial z}+F_{q r}+S_{\text {cond }}+S_{\text {auto }} \\
& +S_{\text {accr }}+S_{\text {gccn. }}
\end{aligned}
$$

The first three terms on the right-hand side correspond to advection, sedimentation, and the subgrid-scale contribution. The next three terms comprise the microphysical processes of condensation/evaporation, autoconversion, and accretion, defined as in Eq. (8) of KK2000. The GCCN source term $S_{\text {gccn }}$ is defined most generally by our Eq. (10).

Relative to explicit microphysical methods, the KK2000 parameterization tends to overestimate small magnitudes of autoconversion (see Fig. 4a in Wood 2005). To alleviate this tendency of the parameterization to overestimate autoconversion predominantly under situations when the background aerosol concentration is large, we incorporate the threshold function of Liu and Daum (2004), as modified by Wood (2005). This has little direct bearing on the fidelity of the GCCN parameterization itself, but is important for establishing a reasonable, no-GCCN baseline simulation, specifically ensuring that the simulation without GCCN produces very little drizzle.

In the explicit simulation, bin microphysical processes are formulated using prognostic equations for $\mathrm{CCN}$ and liquid drops (19 and 25 bins, respectively), with the method most completely described in Kogan (1991). Initial conditions and large-scale forcings for the LES are based on a case study from the Atlantic Stratocumulus Transition Experiment (ASTEX; Albrecht et al. 1995; Duynkerke et al. 1995) case A209 [see Khairoutdinov and Kogan (1999) for details about this case]. Simulations are three-dimensional $(40 \times$ $40 \times 51$ ), with horizontal and vertical grid spacings of 75 and $25 \mathrm{~m}$, respectively, resulting in a domain size of $3 \times 3 \times 1.25 \mathrm{~km}$. The background $\mathrm{CCN}$ concentration is assumed to be $628 \mathrm{~cm}^{-3}$, with the value of $k$ set to 0.49 . We choose a large $\mathrm{CCN}$ concentration because the 

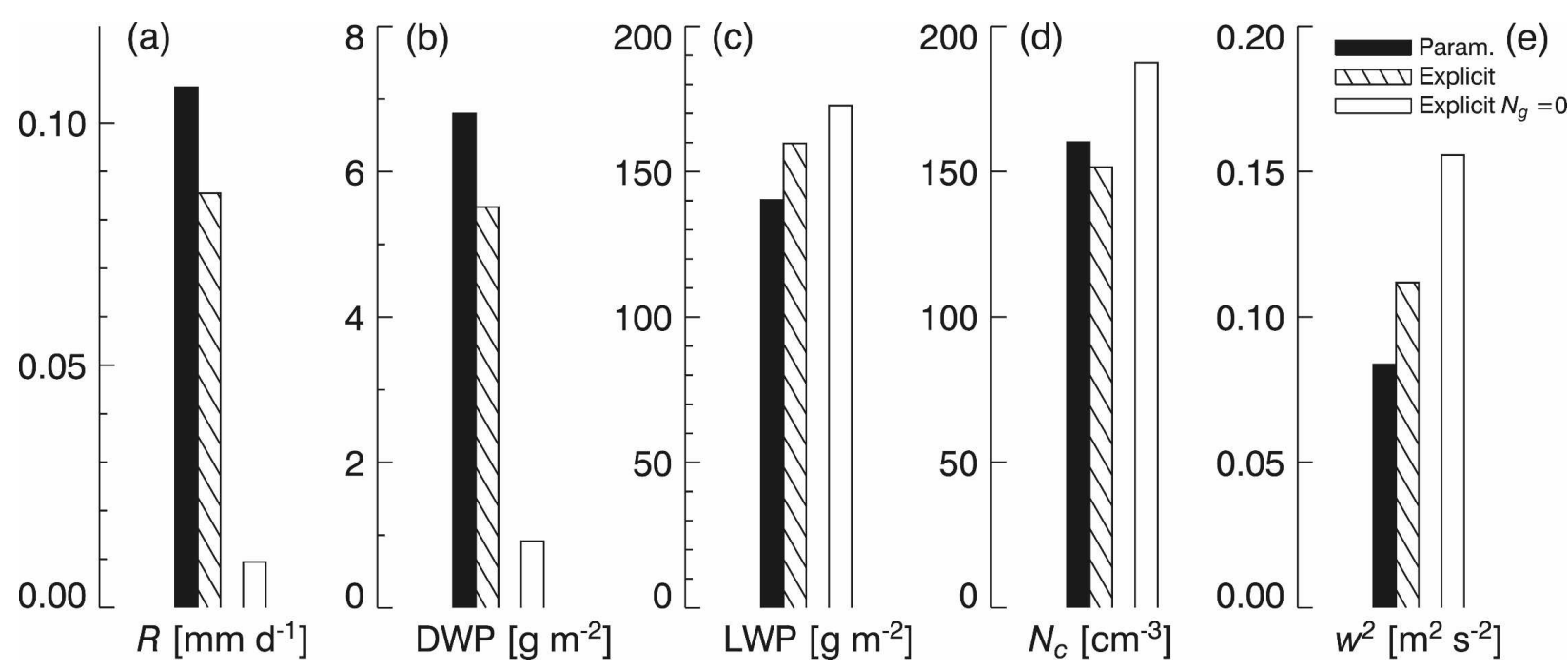

FIG. 1. Comparison of mean (4-6 h) quantities from parameterized GCCN and bin microphysics simulations: (a) surface drizzle rate, (b) drizzle water path, (c) LWP, (d) mean droplet concentration over the cloud layer, and (e) vertical velocity variance averaged over the boundary layer.

effect of adding GCCN is accentuated in environments of high background CCN concentrations (Ghan et al. 1998; Feingold et al. 1999). For the explicit simulation, the background aerosol spectrum is assumed to be lognormal, with total concentration, geometric mean, and geometric standard deviation of $628 \mathrm{~cm}^{-3}, 1.5 \mu \mathrm{m}$, and $0.045 \mu \mathrm{m}$, respectively. These shape parameters are chosen to match the mean droplet concentration of 260 $\mathrm{cm}^{-3}$ in the GCCN simulation. The precise value of the background CCN concentration is not particularly important, so long as it captures the characteristics of a polluted air mass. In section 4 , we contrast the impact of adding various amounts of GCCN to polluted and clean background $\mathrm{CCN}$ environments.

The GCCN concentration $N_{g}$ in Eq. (6) is assumed to be $1360 \mathrm{~L}^{-1}$. This value corresponds to that of the O'Dowd et al. (1997) sea spray "jet" mode observed at the ocean surface in a wind of $10 \mathrm{~m} \mathrm{~s}^{-1}$. The addition of GCCN is formulated as a boundary value problem, with $N_{g}=$ constant at the surface. This makes physical sense, since the most likely source for marine GCCN is the ocean surface. Holding the surface value fixed enables the model's resolved and subgrid-scale dynamics to transport GCCN vertically into the cloud layer. Furthermore, this approach avoids the unphysical "shock" of precipitation when the GCCN is specified as an initial value problem (homogeneously over the entire domain at the initial time). In addition to physical soundness, the boundary value problem avoids the rapid (and we feel, unrealistic) removal of GCCN by cloud processing. For example, when configured as an initial value problem, the $e$-folding decay time for GCCN is 20 min, and after $150 \mathrm{~min}$ the GCCN falls to $1 \%$ of its initial value. Because of the limited dynamical response to increased drizzle after only $20 \mathrm{~min}$, the $e$-folding time scale of GCCN reduction is dictated by simple eddy turnover arguments. The $1 \%$ value is dictated by feedback between drizzle-modulated dynamics and GCCN transport. For the boundary value problem, on the other hand, the subcloud GCCN profiles attain an approximate steady state after an hour of time, indicating that cloud GCCN uptake and processing are in close balance. More generally, of course, the GCCN surface value would be interactively coupled with surface wind speed via a parameterization like that of O'Dowd et al. (1997); however, for simplicity we take the surface GCCN magnitude to be a constant.

The LES is first run for a spinup time of $40 \mathrm{~min}$ in order to establish boundary layer structure. During this time, cloud properties are diagnosed using simple moist saturation adjustment. After the spinup, droplet nucleation proceeds according to $N_{\text {act }}=C S^{k}$, where a value of 0.49 for $k$ equates to droplet concentrations of 285 at $0.2 \%$ supersaturation.

The GCCN parameterization captures the precipitation process reasonably well, as indicated by the surface drizzle rate and drizzle water path (Figs. 1a,b), although the parameterization slightly overestimates these quantities. By way of comparison, drizzle parameters in the bin microphysics simulation without GCCN are much smaller. In this particular case, drizzle acts predominantly as a sink of cloud water, so the stronger drizzle production in the parameterization simulation results in a smaller liquid water path (LWP) relative to the ex- 
plicit simulation (Fig. 1c). Sedimentation of drizzle acting simply to reduce cloud liquid water is not a general rule in stratocumulus but likely particular to the small moisture jump at the cloud top (and hence, the lack of cloud-top entrainment instability mechanisms) associated with the A209 case. Some of the differences in parameters are undoubtedly due to the underlying uncertainty in the KK2000 parameterization (i.e., our estimate of $\sim 20 \%$ uncertainty), in addition to differences arising from the GCCN parameterization itself, but the overall agreement between bulk and explicit models is good. The parameterization is surprisingly good at capturing the reduction in droplet concentration (Fig. 1d) via coalescence processing, and also represents the impact of drizzle on boundary layer dynamics, as evident in the vertical velocity variance. The stable stratification of the boundary layer by drizzle tends to reduce the overall turbulent intensity and promote a more decoupled circulation (in the profile of $w^{2}$, not shown). Drizzle appears to exert little effect on the entrainment rate; the fact that drizzle mostly acts as a simple sink of liquid water (reducing the LWP) is a consequence of this. Weaker turbulence nevertheless producing a similar magnitude of entrainment implies that drizzle processes can influence the entrainment efficiency, which has been suggested by Bretherton et al. (2007).

One caveat relevant to this scheme and bulk microphysical parameterizations in general should be mentioned. In cases of high background aerosol concentration, particularly of small, Aitken-size particles, the presence of GCCN has been shown to suppress supersaturation, leading to a reduction in nucleated droplets (Ghan et al. 1998). Bulk microphysical parameterizations generally employ saturation adjustment methods for condensation and evaporation, rather than explicitly allowing the supersaturated vapor field and the droplets to evolve jointly via the condensational growth equation. For this reason, bulk schemes (including this one) will not fully capture the competing effects of sulfate and GCCN on droplet nucleation. However, the reduction of activated droplets predominantly occurs mostly for very weak updraft velocities $\left(<0.2 \mathrm{~m} \mathrm{~s}^{-1}\right)$ or very large background sulfate concentrations (see Fig. 1 of Ghan et al. 1998), both of which are rather atypical for active convection in the MBL.

\section{Sensitivity to GCCN and ambient $\mathrm{CCN}$ concentrations}

\section{a. Cloud, drizzle, and turbulence}

Numerical simulations based on clean and polluted background conditions are performed in order to evaluate the sensitivity to different concentrations of GCCN.
CCN concentrations are assumed to be 628 (similar to the control experiment discussed above) and $82 \mathrm{~cm}^{-3}$ for the polluted and clean cases, respectively, with the value of $k$ set to 0.49 . For a supersaturation of $0.2 \%$, this equates to nucleated droplet concentrations of 285 and $37 \mathrm{~cm}^{-3}$. The precise values of the background $\mathrm{CCN}$ concentrations are not so important, so long as they provide a relative gauge of characteristic polluted and clean environments.

As in the comparison with the bin microphysics representation, GCCN are specified as a boundary value problem. Simulations with surface concentrations of 30 , 300 , and $1360 \mathrm{~L}^{-1}$ are performed. These GCCN are added to the polluted and clean background $\mathrm{CCN}$ populations of 628 and $82 \mathrm{~cm}^{-3}$, respectively, and are compared to control cases with no GCCN. As expected, the polluted simulations show the greatest sensitivity to the addition of GCCN (Figs. 2a-e). Successively increasing amounts of GCCN added to the polluted background produces effects characteristic of enhanced precipitation formation: larger drizzle rate and drizzle water path (Figs. 2a,b), depleted liquid water path and droplet concentration associated with coalescence processing (Figs. 2c,d), and suppressed boundary layer turbulence (Fig. 2e) arising from drizzle stabilizing the boundary layer. The percentage of liquid water contained in drizzle drops rises from $1.2 \%$ to $4.5 \%$, a result both of increasing drizzle production and decreasing liquid water content from drizzle sedimentation. Similar statistics from Feingold et al. (1999) exhibit a similar trend, with drizzle water percentage ranging from $0 \%$ to $7.9 \%$ over their range of specified GCCN concentrations, although direct comparisons are not particularly insightful, since specific case details and experimental configurations are very different between the two cases.

When added to the clean background $\mathrm{CCN}$, in contrast, GCCN added in any quantity (up to $1360 \mathrm{~L}^{-1}$ ) has little effect, as the cloud is already drizzling significantly (Fig. 2a). (The nonmonotonicity in drizzle rate for the clean simulation series is an artifact of the limited domain size.) The drizzle water percentage is approximately $26 \%$ for all four cases, roughly in accord with the range of $25 \%-32 \%$ found by Feingold et al. (1999). Relative to the polluted background CCN simulations, all of the clean simulations contain more drizzle, less cloud liquid water, and have lower levels of turbulence. This relative sensitivity of cloud and drizzle properties to the addition of GCCN in clean versus background $\mathrm{CCN}$ environments produced by the GCCN parameterization is consistent with previous results employing explicit microphysics (Feingold et al. 1999). 

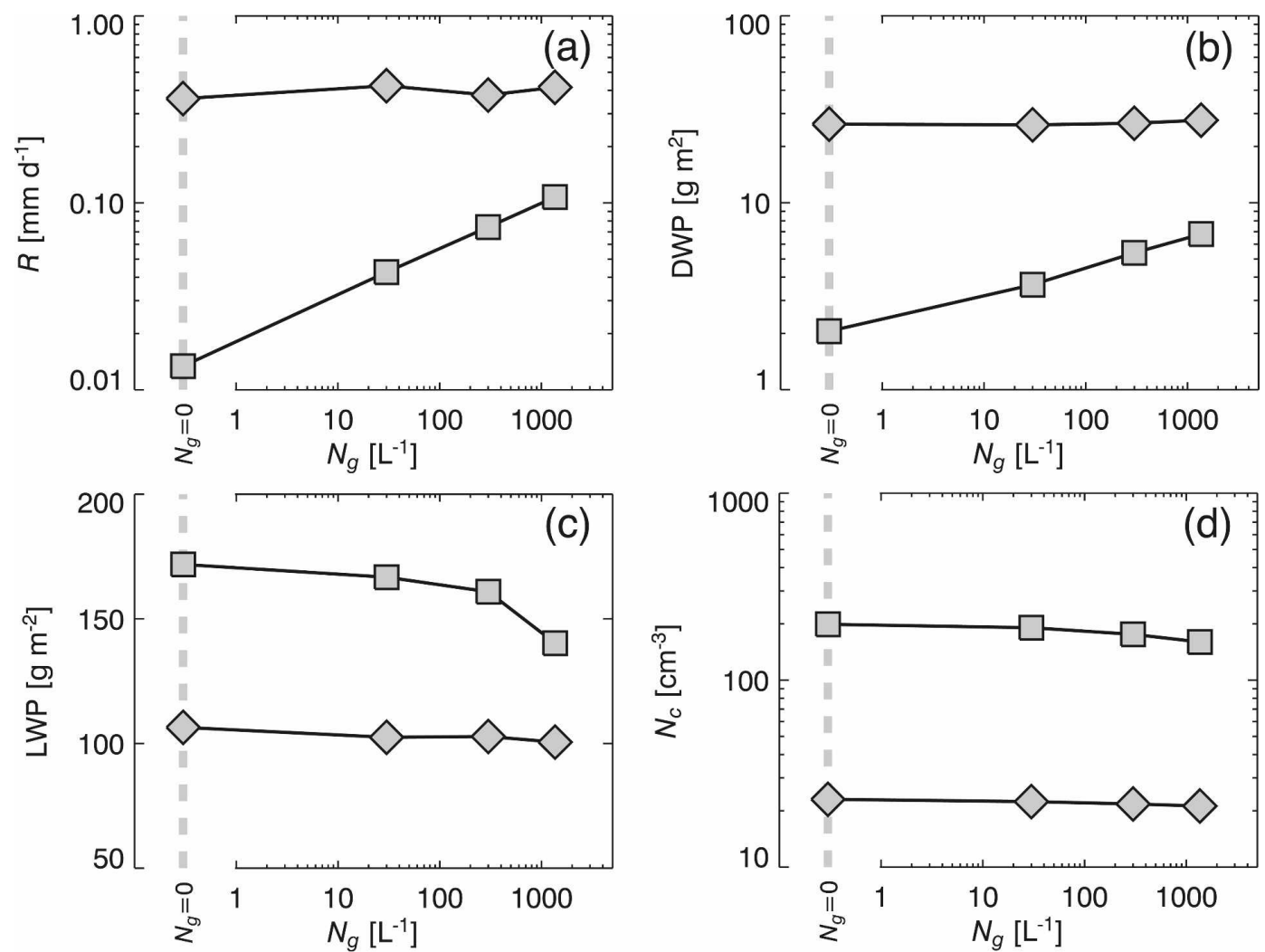

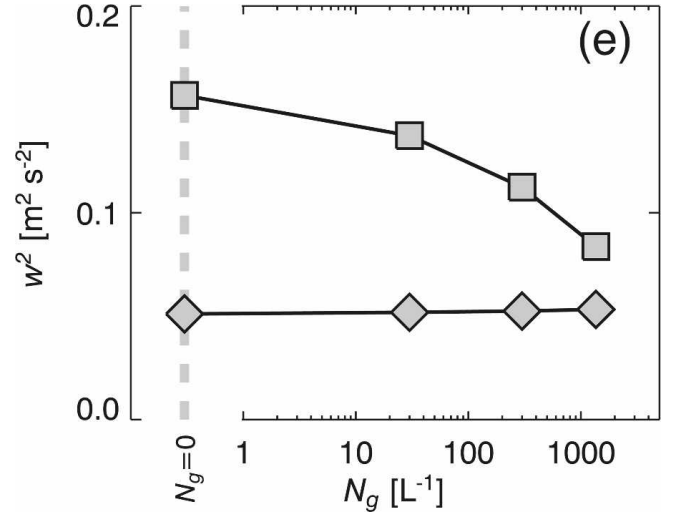

\section{b. Cloud radiative properties}

Our analysis of the influence of GCCN on cloud system radiative properties follows Feingold et al. (1999). Cloud system albedo is a function of optical depth $\tau$,

$$
A=\frac{(1-g) \tau}{2+(1-g) \tau}
$$

where $g$ is the asymmetry parameter $(=0.84)$. Optical depth is calculated by approximating the cloud droplet spectra with a gamma distribution, constrained by the bulk moments of the drop size distribution $\left(q_{c}\right.$ and $\left.N_{c}\right)$.
Aerosol indirect effects are frequently formulated in terms of a sensitivity, called "susceptibility," of albedo to a change in droplet number. Susceptibility $\left(\mathrm{cm}^{3}\right)$ may be approximated as

$$
S=\frac{A(1-A)}{3 N} .
$$

The effect on radiative properties of adding GCCN to background clean and polluted cases is summarized in Fig. 3. Just as for the cloud parameters in Fig. 2, adding GCCN to the clean CCN background has little effect on the magnitude of the cloud optical depth, 

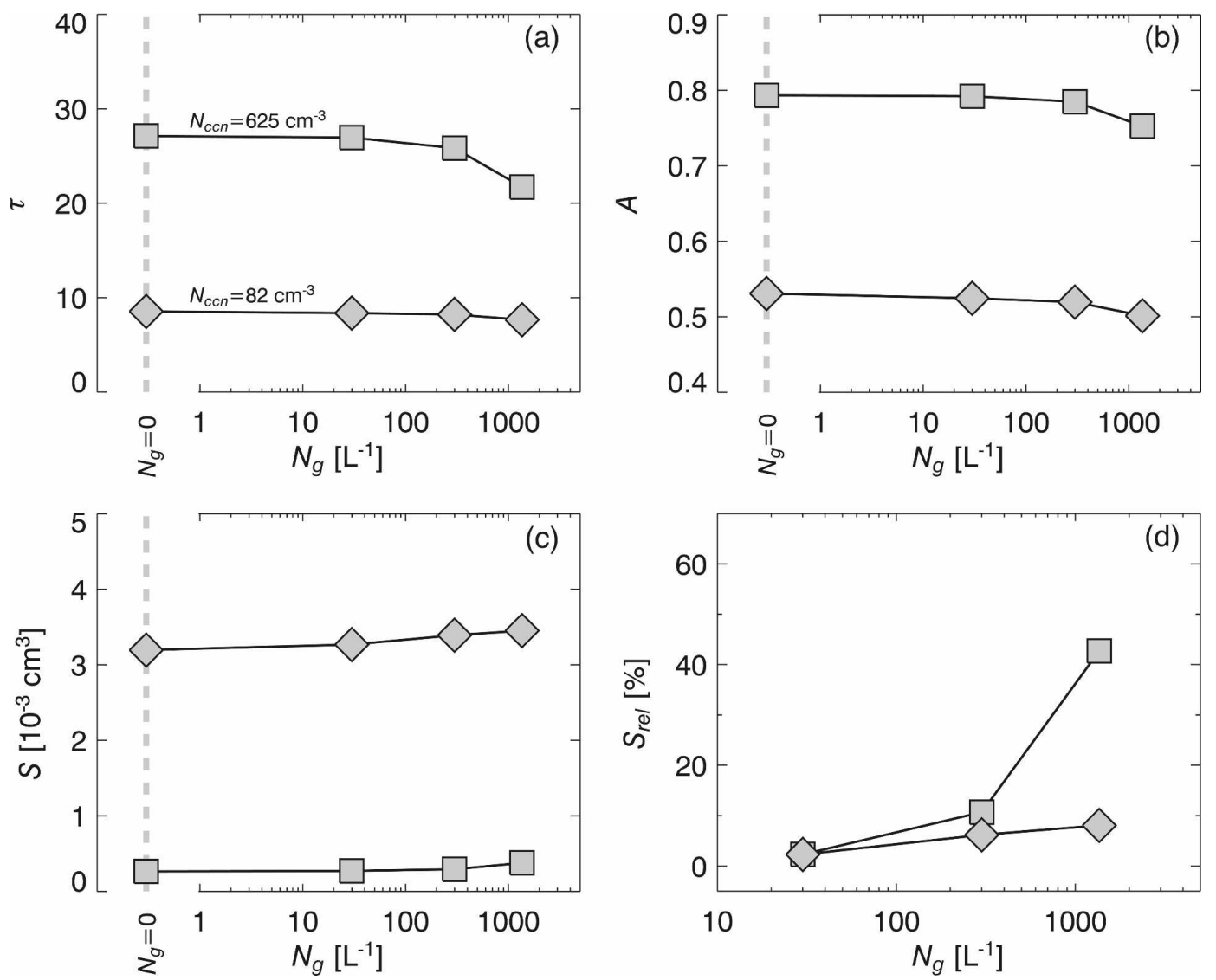

FIG. 3. Hourly mean calculations (3-4 h) of radiative quantities as a function of GCCN concentration for polluted and clean background CCN conditions: (a) optical depth, (b) albedo, (c) susceptibility $[A(1-A) /(3 N)]$, and (d) susceptibility relative to the control simulations without GCCN.

largely because the cloud LWP has already been reduced so much from drizzle. Adding GCCN, however, tends to make them drizzle even more, and owing to the reduced optical depth $(10 \%)$ and further depleted droplet concentration, GCCN leads to a modest (3\%) reduction in albedo. Adding GCCN to the polluted background $\mathrm{CCN}$ results in a more noticeable reduction in optical depth $(21 \%)$ and albedo (4\%). Relative to Feingold et al. (1999), our results appear to exhibit less sensitivity to the addition of GCCN, though as stated before differences between the two approaches preclude a robust direct comparison. In all cases, the reduction in optical depth and albedo arises from a combination of lower cloud liquid water content (from drizzle loss) and a decrease in droplet concentration accompanying drizzle production (collection). Less liquid water spread out over fewer drops decreases the backscatter cross section and the optical depth.

Absolute susceptibility varies little with GCCN concentration but is smaller in the polluted background $\mathrm{CCN}$ environment (Fig. 3c). Equivalent changes in $N$ produce more albedo response in the clean case (small $N$ ) than in the polluted case (large $N$ ). Yet Figs. 2 and 3 plainly demonstrate that cloud properties in the polluted environment are more sensitive to the addition of GCCN. For this reason, susceptibility relative to the control simulations (Fig. 3d) most aptly illustrates the sensitivity of albedo to a change in droplet number. As expected, the relative susceptibility of the polluted case is much greater than that of the clean case and increases with increasing GCCN. Although the experimental configurations are quite different, the relative sensitivity of the cloud optical properties to different GCCN concentrations in clean and polluted environments is generally consistent with Feingold et al. (1999).

\section{Summary}

A new parameterization of giant $\mathrm{CCN}$ suitable for use in bulk microphysical models is presented. The parameterization is based on an assumed GCCN size distribution and detailed calculations of aerosol wetting in 
the subcloud layer. GCCN properties are specified as a concentration $\left(N_{g}\right)$ and the shape parameter of the Junge distribution $(\alpha)$. The parameterization can be easily implemented alongside current bulk microphysical schemes and naturally couples with wind-dependent parameterizations of sea salt flux from the ocean surface (e.g., O'Dowd et al. 1997). When incorporated alongside microphysics schemes that include the ice phase, the parameterization will likely have little effect upon mixed-phase clouds, since in those cases precipitation is ultimately attributable to cold-cloud microphysical processes like vapor growth and accretion of ice-phase particles.

The GCCN parameterization is compared in an LES framework against a size-resolving representation of microphysical processes. The parameterization produces reasonable behavior in cloud, drizzle, and turbulence quantities relative to the bin microphysics method.

Employing the parameterization, cloud and radiative properties exhibit the proper response to changes in GCCN concentration for both polluted and clean background CCN environments. Microphysical and cloud radiative properties exhibit the greatest sensitivity to the addition of GCCN for a large background aerosol concentration, while the addition of GCCN to a clean background environment with preexisting active precipitation results in only a modest change in cloud properties.

The parameterization is best implemented as a boundary value problem with either a constant GCCN value or flux at the surface. This formulation is consistent with the physical source for GCCN (sea spray) and avoids the unphysical burst of precipitation and rapid depletion of GCCN that occurs when configured as an initial value problem. In the boundary value formulation, with the only source of GCCN coming from the surface, after a short time GCCN profiles over the subcloud layer attain a steady state, indicating that GCCN processing by the cloud is in approximate balance with the surface source.

Acknowledgments. We thank two anonymous reviewers whose suggestions improved the clarity of the manuscript. This research was supported by ONR Grants N00014-05-1-0550 and N00014-03-1-0304, by the Office of Science (BER), U.S. Department of Energy Grant DE-FG02-05ER64062, and by NOAA/Office of Oceanic and Atmospheric Research under NOAA/ University of Oklahoma Cooperative Agreement NA17RJ1227, U.S. Department of Commerce. Some of the computing for this project was performed at the OU Supercomputing Center for Education and Research (OSCER) at the University of Oklahoma.

\section{REFERENCES}

Albrecht, B. A., 1989: Aerosols, cloud microphysics, and fractional cloudiness. Science, 245, 1227-1230.

, C.S. Bretherton, D. Johnson, W. H. Schubert, and A.S. Frisch, 1995: The Atlantic statocumulus experimentASTEX. Bull. Amer. Meteor. Soc., 76, 889-904.

Beard, K. V., and H. T. Ochs III, 1993: Warm-rain initiation: An overview of microphysical mechanisms. J. Appl. Meteor., 32, 608-625.

Blyth, A. M., S. G. Lasher-Trapp, W. A. Cooper, C. A. Knight, and J. Latham, 2003: The role of giant and ultragiant nuclei in the formation of early radar echoes in warm cumulus clouds. J. Atmos. Sci., 60, 2557-2572.

Bretherton, C. S., P. N. Blossey, and J. Uchida, 2007: Cloud droplet sedimentation, entrainment efficiency, and subtropical stratocumulus albedo. Geophys. Res. Lett., 34, L03813, doi:10.1029/2006GL027648.

Cooper, W. A., R. T. Bruintjes, and G. K. Mather, 1997: Calculations pertaining to hygroscopic seeding with flares. J. Appl. Meteor., 36, 1449-1469.

Duynkerke, P. G., H. Zhang, and P. J. Jonker, 1995: Microphysical and turbulent structure of nocturnal stratocumulus as observed during ASTEX. J. Atmos. Sci., 52, 2763-2777.

Feingold, G., W. R. Cotton, S. M. Kreidenweis, and J. T. Davis, 1999: The impact of giant cloud condensation nuclei on drizzle formation in stratocumulus: Implications for cloud radiative properties. J. Atmos. Sci., 56, 4100-4117.

Ghan, S. J., G. Guzman, and H. Abdul-Razzak, 1998: Competition between sea salt and sulfate particles as cloud condensation nuclei. J. Atmos. Sci., 55, 3340-3347.

Ivanova, E. T., Y. L. Kogan, I. P. Mazin, and M. S. Permyakov, 1977: Method of parameterizing the condensation process of droplet growth in numerical models. Izv. Akad. Sci. USSR Atmos. Ocean Phys., 13, 821-826.

Johnson, D. B., 1982: The role of giant and ultragiant aerosol particles in warm rain initiation. J. Atmos. Sci., 39, 448-460.

Junge, C. E., 1972: Our knowledge of the physico-chemistry of aerosols in the undisturbed marine environment. J. Geophys. Res., 77, 5183-5200.

Kessler, E., 1969: On the Distribution and Continuity of Water Substance in Atmospheric Circulations. Meteor. Monogr., No. 32, Amer. Meteor. Soc., 84 pp.

Khairoutdinov, M. F., and Y. L. Kogan, 1999: A large eddy simulation model with explicit microphysics: Validation against aircraft observations of a stratocumulus-topped boundary layer. J. Atmos. Sci., 56, 2115-2131.

$\longrightarrow$, and - 2000: A new cloud physics parameterization for large-eddy simulation models of marine stratocumulus. Mon. Wea. Rev., 128, 229-243.

Kogan, Y. L., 1991: The simulation of a convective cloud in a 3D model with explicit microphysics. Part I: Model description and sensitivity experiments. J. Atmos. Sci., 48, 1160-1188.

Liu, Y., and P. H. Daum, 2004: Parameterization of the autoconversion process. Part I: Analytical formulation of the Kesslertype parameterizations. J. Atmos. Sci., 61, 2923-2940.

,-- , and R. McGraw, 2004: An analytical expression for predicting the critical radius in the autoconversion parameterization. Geophys. Res. Lett., 31, L06121, doi:10.1029/ 2003 GL019117.

Mordy, W., 1959: Computations of the growth by condensation of a population of cloud drops. Tellus, 11, 16-44.

O'Dowd, C. D., M. H. Smith, I. E. Consterdine, and J. A. Lowe, 
1997: Marine aerosol, sea-salt, and the marine sulphur cycle: A short review. Atmos. Environ., 31, 73-80.

Paluch, I. R., and D. H. Lenschow, 1991: Stratiform cloud formation in the marine boundary layer. J. Atmos. Sci., 48, 21412158.

Saleeby, S. M., and W. R. Cotton, 2004: A large-droplet mode and prognostic number concentration of cloud droplets in the Colorado state regional atmospheric modeling system (RAMS). Part I: Module descriptions and supercell test simulations. J. Appl. Meteor., 43, 182-195.
Stevens, B., W. R. Cotton, G. Feingold, and C.-H. Moeng, 1998: Large-eddy simulations of strongly precipitating, shallow, stratocumulus-topped boundary layers. J. Atmos. Sci., 55, 3616-3638.

Tripoli, G. J., and W. R. Cotton, 1980: A numerical investigation of several factors contributing to the observed variable intensity of deep convection over South Florida. J. Appl. Meteor., 19, 1037-1063.

Wood, R., 2005: Drizzle in stratiform boundary layer clouds. Part II: Microphysical aspects. J. Atmos. Sci., 62, 3034-3050. 